\title{
MicroRNA-155 Suppresses Mesangial Cell Proliferation and TGF- $\beta 1$ Production via Inhibiting CXCR5-ERK Signaling Pathway in Lupus Nephritis
}

\author{
Jie Kong, ${ }^{1}$ Liuxia Li, ${ }^{1}$ Zhimin Lu, ${ }^{1}$ Jiamin Song, ${ }^{1}$ Jiaxin Yan, ${ }^{1}$ Junling Yang, ${ }^{2}$ Zhifeng Gu, ${ }^{1,2}$ and \\ Zhanyun $\mathbf{D a}^{1,3}$
}

Abstract - Increasing evidence shows miR-155 plays an important role in regulating inflammatory processes in systemic lupus erythematosus (SLE), especially in lupus nephritis (LN). Because the chemokine CXCL13 is implicated in the pathogenesis of LN, here we examined whether miR-155 can modulate the activity of CXCL13 or its receptor CXCR5. We determined the expression of CXCL13 in normal and MRL/lpr mice and found elevated levels of CXCL13 in the kidneys of MRL/lpr mice compared with normal kidneys. Besides, CXCL13 expression was mainly detected in the glomerulus, specifically to mesangial areas. We then transfected a miR-155 mimic in human renal mesangial cells (HRMCs) to overexpress miR155 and detected decreased protein levels of CXCR 5 by western blot analysis. Transfection of the miR-155 mimic into CXCL13-treated HRMCs resulted in a significantly reduced proliferation rate of HRMCs as measured by the cell-counting assay and flow cytometry. Moreover, increased intracellular miR-155 also led to decreased phosphorylation of ERK and TGF- $\beta 1$ production. Together, these results revealed that miR-155 may play a role in the pathogenesis of LN.

KEY WORDS: CXCL13; miR-155; mesangial cell; lupus nephritis.

\section{INTRODUCTION}

Systemic lupus erythematosus (SLE) is an autoimmune disease with immune complexes formation and deposition in multiple organs, among which, the kidney is one of the major target organs $[12,34]$. At least 30 to $60 \%$

Jie Kong and Liuxia Li contributed equally to this work.

${ }^{1}$ Department of Rheumatology, Affiliated Hospital of Nantong University, No. 20, XiSi Road, Nantong, 226001, Jiangsu Province, People's Republic of China

${ }^{2}$ Research Center of Clinical Medicine, Affiliated Hospital of Nantong University, No. 20, XiSi Road, Nantong, 226001, Jiangsu Province, People's Republic of China

${ }^{3}$ To whom correspondence should be addressed at Department of Rheumatology, Affiliated Hospital of Nantong University, No. 20, XiSi Road, Nantong, 226001, Jiangsu Province, People's Republic of China. E-mail: dazhanyun@163.com
SLE patients have lupus nephritis (LN), and almost all patients have pathological kidney involvement $[2,6]$. Notably, $10 \%$ of LN patients progress to end-stage renal disease (ESRD) [16]. Consequently, LN is one of the leading causes of death in SLE $[7,26]$. In recent years, early diagnosis, standardized treatment, and new immunosuppressive agents such as mycophenolate mofetil [19], anti-CD20 monoclonal antibody, belimumab [11], and other drugs have significantly improved the prognosis of LN. However, the mortality rate within 1-5 years in severe refractory LN patients is still high [1]. Therefore, it is of great importance to explore the pathogenesis of LN and search for new therapeutic targets.

MicroRNAs (miRNAs) are newly discovered noncoding RNA molecules composed of $\sim 22$ nucleotides, and they can regulate the expression of target genes by binding to the $3^{\prime}$ untranslated region of the mRNA (3' 
UTR) [31]. MiRNAs play important roles in various physiological and pathological processes such as cell proliferation, differentiation, and apoptosis by downregulating the expression of target genes $[4,15]$. MiR-155 is a member of the miRNA superfamily that mediates innate and adaptive immune responses and plays an important role in regulating blood cell generation, inflammation, and immune responses [18]. Previous studies used bioinformatic analysis for miRNA target prediction of genes and found that miR155 was involved in the ERK/MAPK signaling pathway [23]. Moreover, miR-155 has been reported to inhibit the secretion of inflammatory factors by downregulating the ERK/MAPK signaling pathway [3]. Recent studies found that the serum levels of miR-155 were significantly lower in SLE patients than those in healthy individuals. Thus, miR-155 may serve as an additional serological marker for SLE [32].

Here, we examined the effects of increased intracellular levels of miR-155 activity on the proliferation of human renal mesangial cells (HRMCs) and the expression of the CXCR5, which were inhibited by the overexpression of miR-155. Moreover, both protein levels of p-ERK and transforming growth factor $\beta 1$ (TGF- $\beta 1$ ) production were also reduced in miR-155-overexpressed HRMCs. Therefore, our results suggest that miR-155 can suppress CXCL13-induced proliferation of HRMCs in LN by downregulating the CXCR5-ERK signaling pathway.

\section{MATERIALS AND METHODS}

\section{Cell Culture}

HRMCs were obtained from JENNIO Biological Technology and cultured in RPMI 1640 medium supplemented with $10 \%$ fetal bovine serum (FBS) and $1 \%$ penicillin/streptomycin, at $37{ }^{\circ} \mathrm{C}$ in a $5 \% \mathrm{CO}_{2}$-humidified atmosphere.

\section{Cell Transfection}

HRMCs were placed into 6-well plates at $37{ }^{\circ} \mathrm{C}$ under $5 \% \mathrm{CO}_{2}$ for $24 \mathrm{~h}$ before transfection. The miR-155 mimic (Biomics Biotech, China) was transfected into cells using Lipofectamine 2000 (Invitrogen by Life Technologies, USA) at 50\% confluency. We used siRNA (Biomics Biotech, China) to silence the expression of CXCR5 in HRMCs. The optimal concentration for transfection was established at $100 \mathrm{nmol} / \mathrm{mL}$ siRNA. The transfection medium contained no FBS or penicillin/streptomycin. After 4-6 h of transfection, the medium was replaced by RPMI
1640 with $10 \%$ FBS, and the cells were then incubated at $37{ }^{\circ} \mathrm{C}$ with $5 \% \mathrm{CO}_{2}$ for $48-72 \mathrm{~h}$. Total protein was collected.

\section{Cell Proliferation Assay}

Cell proliferation was monitored using the Cell Counting Kit-8 (Sangon Biotech, Shanghai, China) according to the manufacturer's instructions. Aliquots of $100 \mu \mathrm{L}$ cell suspension were plated into 96-well plates at $1 \times 10^{3}$ cells per well and cultured in the growth medium for $24 \mathrm{~h}$. Cells were then treated with $0.5 \mathrm{ng} / \mathrm{mL}$ recombinant human CXCL13 (R\&D Systems, USA). HRMCs were transfected with either $50 \mathrm{nmol} / \mathrm{mL}$ miR$155 \mathrm{mimic}$ or $100 \mathrm{nmol} / \mathrm{mL}$ siRNA in RPMI 1640 (without serum or antibiotics). The number of viable cells was assessed at 0,6,12, 24, and $36 \mathrm{~h}$ after treatment. Each well was added with $10 \mu \mathrm{L}$ CCK-8 solution, incubated for $2.5 \mathrm{~h}$ in the dark, and measured the absorbance at $450 \mathrm{~nm}$ using a microplate reader (BioTek, USA).

\section{Flow Cytometric Analysis}

Cell cycles of HRMCs with CXCL13 treatment or transfection with siRNA or miRNA were determined by flow cytometry. Cells were digested in $500 \mu \mathrm{L} 0.05 \%$ trypsin-EDTA for 5-7 min and added 500 $\mu \mathrm{L}$ RPMI 1640 supplemented with $10 \%$ FBS to inactivate trypsin. Cells were then centrifuged at $1200 \mathrm{rpm}$ for $5 \mathrm{~min}$, washed and resuspended in cold PBS twice, and incubated at $20{ }^{\circ} \mathrm{C}$ in $70 \%$ ethanol for at least $24 \mathrm{~h}$. They were then permeabilized with $200 \mu \mathrm{L}$ PBS containing $1 \%$ Triton X100 for $10 \mathrm{~min}$. Finally, the cells were resuspended in $500 \mu \mathrm{L}$ propidium iodide (PI)/RNase staining buffer (BD Pharmingen, USA) for $15 \mathrm{~min}$ in the dark and analyzed by flow cytometry. MFLT32 Soft was used to calculate the fraction of cells in $\mathrm{S}$ phase.

\section{Immunohistochemistry}

For immunohistochemistry, kidney samples were fixed in $4 \%$ buffered paraformaldehyde and embedded in paraffin. This was followed by deparaffinizing the samples twice with xylene for $15 \mathrm{~min}$ and rehydrating in descending grades of alcohol (100-70\%). Then, sections were heated in a microwave oven at $100{ }^{\circ} \mathrm{C}$ with $10 \mathrm{mM}$ citrate buffer ( $\mathrm{pH} \mathrm{6.0)}$ ) for $10 \mathrm{~min}$ to retrieve the antigen and washed three times with PBS. Endogenous peroxidase was inhibited by incubation in $3 \% \mathrm{H}_{2} \mathrm{O}_{2}$ for $10 \mathrm{~min}$. Samples were then incubated with anti-CXCL13 (Abcam, USA) as primary antibody at $4{ }^{\circ} \mathrm{C}$ overnight, followed by 
a subsequent incubation with peroxidase-labeled goat antirabbit $\lg \mathrm{G}$ for $20 \mathrm{~min}$ at room temperature. Peroxidase activity was detected using diaminobenzidine (DAB) as substrate, and the nuclei were counterstained with hematoxylin. MRL/MPJ mice were used as control. The study was approved by the Ethics Committees of Affiliated Hospital of Nantong University. The approval number is 2017 L096.

\section{Immunofluorescent Staining of Kidney Tissue}

The expression and localization of CXCL13 in renal tissues were determined by immunofluorescence assay. In brief, kidney samples were deparaffinized, rehydrated, and antigen-retrieved as described. Double-staining was achieved by incubating the specimen with anti-CXCL13 (Abcam, USA) and anti-collagen 4 (Abcam, USA) as primary antibodies at $4{ }^{\circ} \mathrm{C}$ overnight, followed by incubation with daylight594 goat anti-rabbit IgG (Abbkine, USA) and daylight488 goat anti-mouse $\operatorname{IgG}$ (Abbkine, USA) as secondary antibodies at room temperature for $2 \mathrm{~h}$ in the dark. After three washes with $1 \times$ PBS in the dark, the samples were mounted with 4',6-diamidino-2phenylindole mounting medium (DAPI, Beyotime Biotechnology, China) for $10 \mathrm{~min}$. Images were captured using an immunofluorescence microscope (Olympus, USA).

\section{Immunofluorescent Staining in Cell Culture}

Cells were first fixed in $4 \%$ paraformaldehyde for 40 min, washed three times with PBS, and then permeabilized with 1\% TritonX-100 (Beyotime Biotechnology, China) at $4{ }^{\circ} \mathrm{C}$ for $10 \mathrm{~min}$, before incubating with primary antibody anti-phospho-p44/42 MAPK(ERK1/2) (Cell Signaling Technology, USA), at $4{ }^{\circ} \mathrm{C}$ overnight. After washing with $1 \times$ PBS, cells were incubated with daylight594 goat anti-rabbit IgG (Abbkine, USA) for $2 \mathrm{~h}$ in the dark. The nuclei were stained with DAPI (Beyotime Biotechnology, China). Thereafter, the coverslips were viewed under an immunofluorescence microscope (Olympus, USA).

\section{Enzyme-Linked Immunosorbent Assay}

The concentrations of TGF- $\beta 1$, MCP-1, and IL- 1 in the cell culture supernatants were measured using an ELISA kit (R\&D Systems, USA) according to the directions of the manufacturer.

\section{Western Blot}

Cells were lysed in $5 \times$ SDS-PAGE Sample Loading Buffer, $100 \mathrm{mM}$ RIPA Lysis Buffer, and $1 \mathrm{mM}$ PMSF
(Beyotime Biotechnology, China) and subsequently heated to $95{ }^{\circ} \mathrm{C}$ for $5 \mathrm{~min}$. Total protein was quantified by BCA (Beyotime Biotechnology, China). The proteins were transferred to a polyvinylidene difluoride membrane (PVDF) using a Mini Trans-Blot apparatus (Bio-Rad, Hercules, USA). The filters were incubated in TBST with $5 \%$ nonfat dry milk for $1-2 \mathrm{~h}$ at room temperature and then at $4{ }^{\circ} \mathrm{C}$ overnight with anti-CXCR5 (Abcam, USA), and antiphospho-p44/42 MAPK(ERK1/2) (Cell Signaling Technology, USA) or anti-p44/42 MAPK(ERK1/2) (Cell Signaling Technology, USA) and GAPDH antibody (Proteintech, USA) and then washed with TBST. After further incubation with secondary antibody conjugated with horseradish peroxidase (HRP) for $1 \mathrm{~h}$ at room temperature, relative expression levels of protein were quantified using Quantity One software by ECL.

\section{Statistical Analysis}

Data were collected from three independent experiments and shown as the means \pm standard deviation (SD). Statistical analysis was performed using GraphPad Prism. Oneway analysis of variance (ANOVA) was used to indicate the differences. $p<0.05$ was defined as significant.

\section{RESULTS}

\section{CXCL13 Is Highly Expressed in the Glomerular of LN Patients}

The B lymphocyte chemoattractant BLC/CXCL13 is a CXC chemokine which involved in kidney pathogenesis of LN [10]. Our previous study indicated that the serum concentrations of CXCL13 were significantly higher in LN patients than those in healthy controls [5]. To determine whether the expression of CXCL13 is upregulated in the renal tissue of LN patients, we detected the level of CXCL13 in paraffin-embedded kidney tissues from normal and MRL/lpr by immunohistochemisty and immunofluorescent (CXCL13 and collagen 4) staining. We found that the expression levels of CXCL13 in the kidneys of $\mathrm{MRL} / \mathrm{lpr}$ mice were significantly increased in comparison to normal mice (Fig. 1 and Fig. 2). Moreover, CXCL13 was located mainly in glomerular regions; however, the staining of CXCL13 was much weaker in renal tubule and renal interstitium regions (Fig. 2e-h). The colocalization of CXCL13 (red stain) and collagen 4 (green stain) was observed in the mesangial areas (Fig. 2d, h). Thus, there is a possibility that the mesangial cells are responsible for the high expression of CXCL13. 

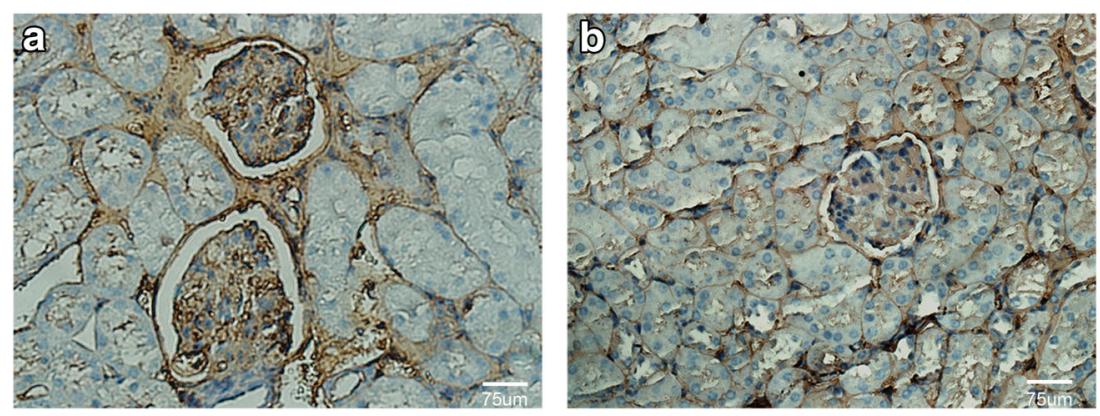

Fig. 1. Detection of CXCL13 in mouse kidney tissues using immunochemical staining. Staining of CXCL13 in MRL/lpr mice (a) and control (b). Original magnification, $\times 400$.

\section{Increasing the Abundance of miR-155 Reduces CXCL13-Induced Proliferation of HRMCs}

The level of miR-155 in the serum of SLE patients was significantly lower than that of healthy individuals [32], suggesting a protective role of miR-155 in reducing the proliferation of mesangial cells, endothelial cells, or podocytes in $\mathrm{LN}$ patients. We raised the abundance of miR155 in HRMCs by transfection with a miR-155 mimic and then used the CCK8 kit to examine the effects of the elevated miR-155 on the growth of the cells. The HRMCs transfected with a miR-155 mimic or transfected with siRNA to silence CXCR5 were then treated with or without CXCL13, and cell variability was then detected after 0,6 , 12,24 , and $36 \mathrm{~h}$.

We observed that CXCL13 treatment could significantly increase the cell proliferation in HRMCs (Fig. 3a); however, overexpression of miR-155 could significantly abrogate CXCL13-stimulated cell proliferation. No significant difference in the growth rates was observed between non-treated cells and miR-155 mimic-treated cells (Fig. 3a). Moreover, transfection of siRNA to silence CXCR5 could also reverse CXCL13-stimulated HMRC proliferation (Fig. 3b). The results led us to hypothesize that miR-155 may decrease the ability of CXCL13-treated HRMCs to proliferate by downregulating CXCR5.

Flow cytometric analysis was used to determine the fraction of cells in S phase (Fig. 3c-g) during cell proliferation. Consistently, we found that CXCL13 could significantly increase the percentage of cells in $\mathrm{S}$ phase compared with non-treated controls $(76.09 \pm 1.44$ vs. $68.88 \pm 0.70)$ (Fig. 3e, c). Increasing the abundance of miR-155 in CXCL13-treated HRMCs reduced the percentage of S phase from $76.09 \pm 1.44$ to $62.03 \pm 0.3$, which was similar to that observed after CXCR5-silencing $(65.46 \pm 0.05)$, see Fig. 3(e-g) and Table 1. No significant difference in the growth rates was observed between non-treated cells and miR-155 mimic-treated cells (Fig. 3c, d). Briefly, the
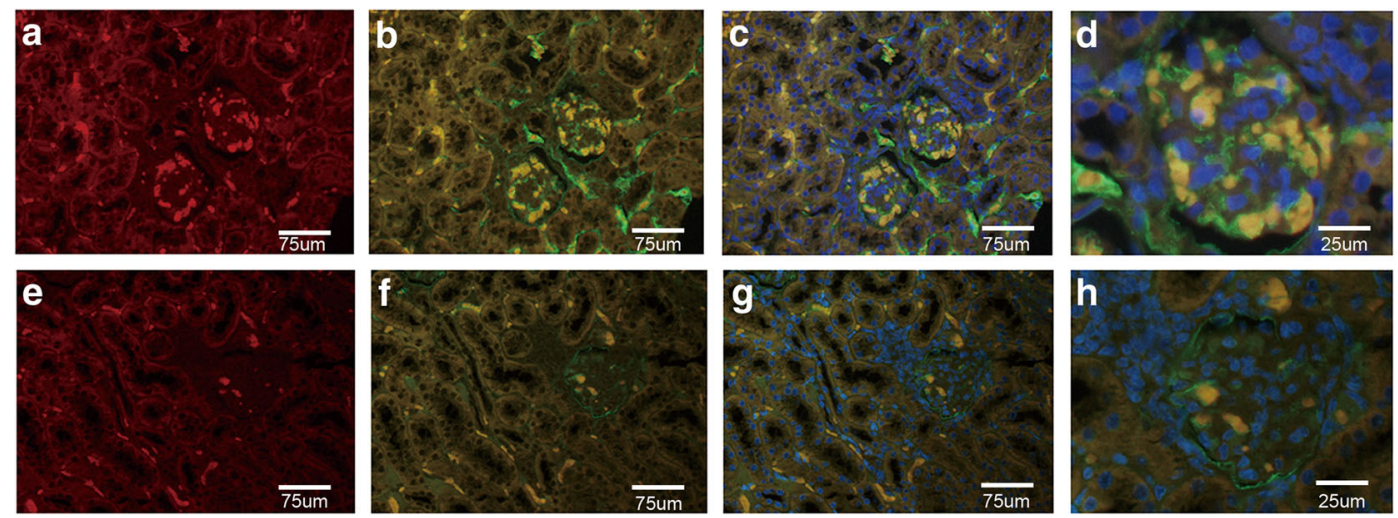

Fig. 2. Detection of CXCL13 and collagen 4 expression in renal tissues by immunofluorescent microscopy. Colocalization of CXCL13 (red) and collagen 4 (green) merged to yellow in images. (a-d) Low expression of CXCL13 in glomerulus, renal tubules, and renal interstitium in control mice. (e-h) High expression of CXCL13 in glomerulus of MRL/lpr mice. $(\mathbf{d}, \mathbf{h})$ The indicated areas in Figs. C and D showing the location of CXCL13 and collagen 4 in the glomerulus. Original magnification, $\times 400$. 
a

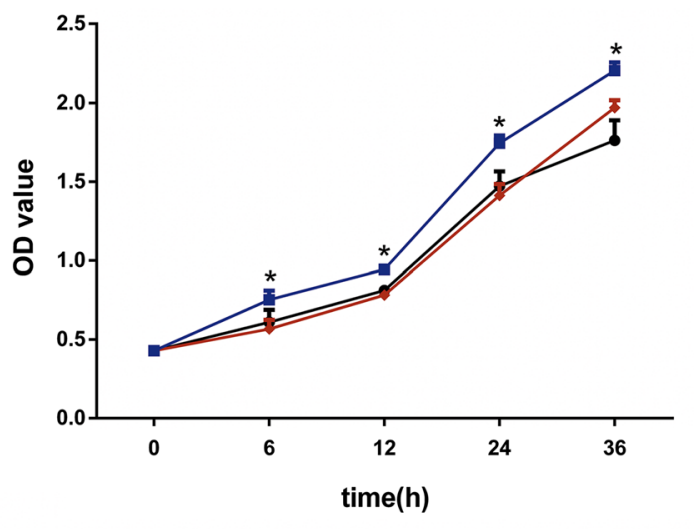

- CXCL13+NC $\rightarrow \mathrm{NC}$

C

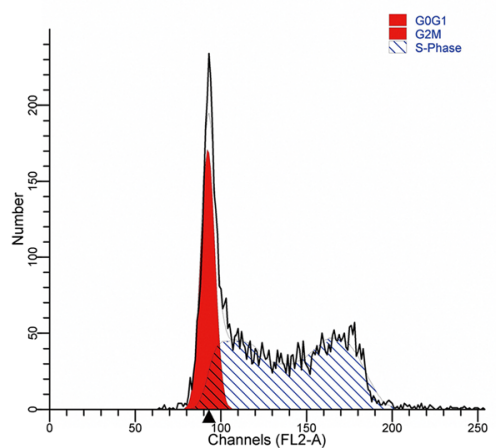

NC

e

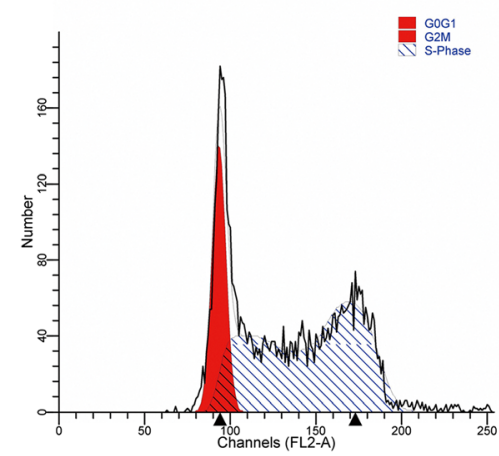

CXCL13+NC b

CCK8

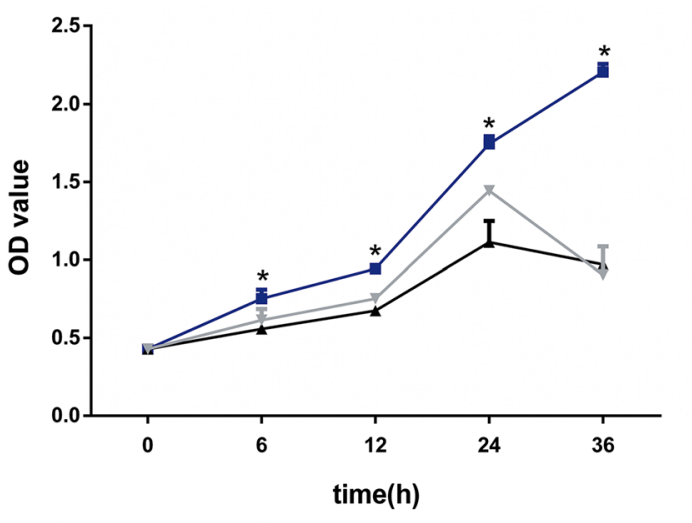

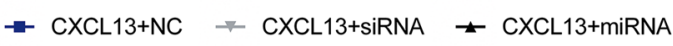

d

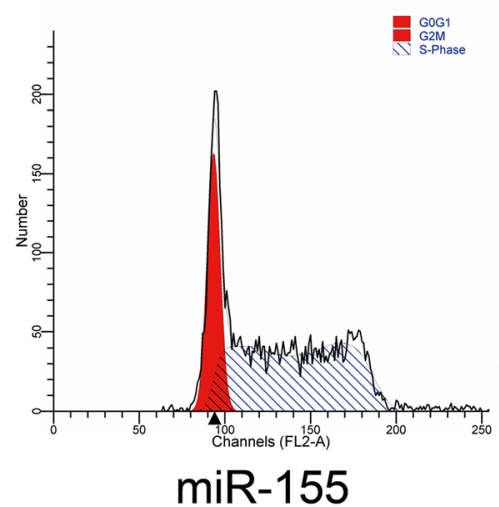

f

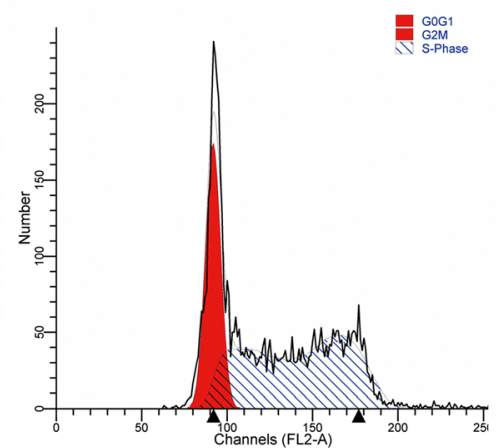

CXCL13+siRNA g

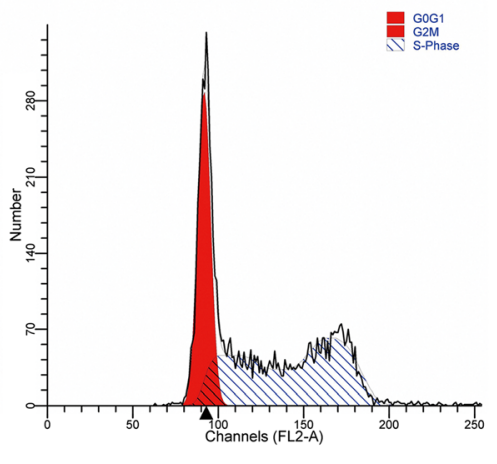

Fig. 3. Effect of transfection with a miR-155 mimic on the proliferation rate of HRMCs stimulated by CXCL13: analysis with the CCK8 kit and flow cytometry. Optimal concentrations of CXCL13 $(0.5 \mathrm{ng} / \mathrm{mL})$, miR-155 mimic $(50 \mathrm{nmol} / \mathrm{mL})$, and siRNA for the silencing of CXCR5 (100 nmol/mL), determined previously, were used in the transfection experiments. (a) Proliferation of CXCL13-treated negative control (NC) (blue line); NC (black line); or miR-155 mimic-transfected cells (red line). (b) Proliferation of NC treated with $0.5 \mathrm{ng} / \mathrm{mL}$ CXCL13 (blue line); or CXCL13-treated cells transfected with siRNA (gray line); or CXCL13-treated cells transfected with a miR-155 mimic (black line). (c-g) Fractions of cells in S phase in HRMCs from the above five groups were analyzed by flow cytometry. Statistical analyses were performed by ANOVA followed by Dunnett's test. * $p<0.05$. 
Table 1. Ratio of HRMCs in S Phase From Different Groups

\begin{tabular}{lll}
\hline & S phase (mean $\pm \mathrm{SD})$ & $* p$ \\
\hline NC\% & $68.88 \pm 0.70$ & - \\
MiR-155\% & $68.10 \pm 1.57$ & $>0.05($ versus $\mathrm{NC} \%)$ \\
CXCL13 + NC\% & $76.09 \pm 1.44$ & $<0.05($ versus $\mathrm{NC} \%)$ \\
CXCL13 + MiR-155\% & $62.03 \pm 0.30$ & $<0.05($ versus CXCL13 + NC\%) \\
CXCL13 + siRNA\% & $65.46 \pm 0.05$ & $<0.05$ (versus CXCL13 + NC\%) \\
\hline
\end{tabular}

Statistical analyses were performed by ANOVA followed by Dunnett's test. $* p<0.05$ was considered significant

results of the CCK8 tests and flow cytometric analysis showed that miR-155 could reduce the proliferation of HRMCs by downregulating CXCR5.

\section{MiR-155 Reduces the Expression of CXCR5}

CXCR5 is a unique receptor for CXCL13 [25]. To validate whether CXCR5 was a target gene of miR-155 in HRMCs, we transfected miR-155 mimic into HRMCs. Overexpression of miR-155 in HRMCs resulted in a significant decreased expression of CXCR5. Moreover, a

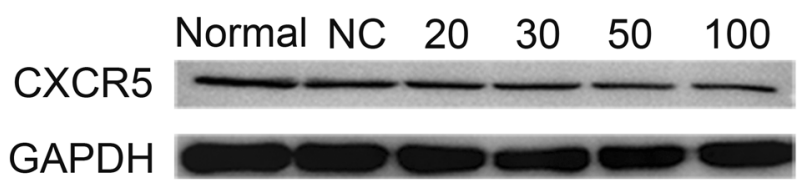

a

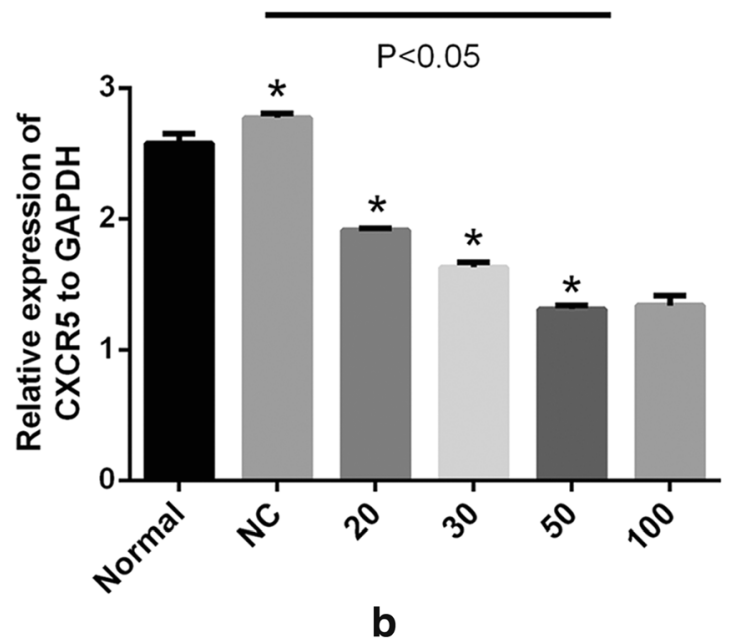

Fig. 4. Western blot assay of the protein levels of CXCR5 in HRMCs transfected with a miR-155 mimic. (a) Cells were transfected with a miR$155 \mathrm{mimic}$ at $20 \mathrm{nmol} / \mathrm{mL}, 30 \mathrm{nmol} / \mathrm{mL}, 50 \mathrm{nmol} / \mathrm{mL}$, and $100 \mathrm{nmol} / \mathrm{mL}$, and the levels of CXCR5 protein were determined. (b) Relative expression of CXCR5 to GAPDH was calculated. Statistical analyses were performed by ANOVA followed by Dunnett's test. $* p<0.05$. concentration of $50 \mathrm{nmol} / \mathrm{mL}$ miR-155 mimic could dramatically inhibit the expression of CXCR5 (Fig. 4).

\section{MiR-155 Reduces ERK Tyrosine Phosphorylation}

Phosphorylation of ERK is associated with increased cell proliferation rate [29]. To investigate whether increasing the abundance of miR-155 would reduce ERK phosphorylation, we transfected the miR-155 mimic into HRMCs and then detected the level of ERK phosphorylation using western blot and immunofluorescent staining (Fig. 5a-c).

We found that CXCL13 could significantly increase the protein level of phosphorylated ERK in miR-155 mimic-transfected cells, and increased miR-155 can reduce ERK tyrosine phosphorylation (Fig. 5a, b). We also investigated whether the inhibition of $\mathrm{p}$-ERK formation by miR155 was mediated by CXCR5. We used a siRNA-specific for silencing the expression of CXCR5 and found a marked decrease in the level of p-ERK (Fig. 5a, b). The decrease in phosphorylation of ERK was similar to that obtained by increasing the abundance of miR-155. Thus, the results are consistent with miR-155 reducing the level of p-ERK by downregulating CXCR5.

\section{MiR-155 Suppresses TGF- $\beta 1$ Production by HRMCs}

TGF- $\beta 1$ has the ability to stimulate mesangial cells and tubular epithelial cells to undergo myofibroblastic activation or transition in vitro [33]. In this study, we measured the concentration of TGF- $\beta 1$, MCP-1, and IL-1 in the supernatants of HRMC culture system using ELISA. Upon treating HRMCs with CXCL13, the concentration of TGF- $\beta 1$ increased. Moreover, the concentration of TGF$\beta 1$ decreased when we increased the abundance of miR155 in HRMCs (Fig. 5d). Besides, the concentration of MCP-1 and IL-1 showed no significant change in the supernatant of CXCL13-treated HRMCs. 


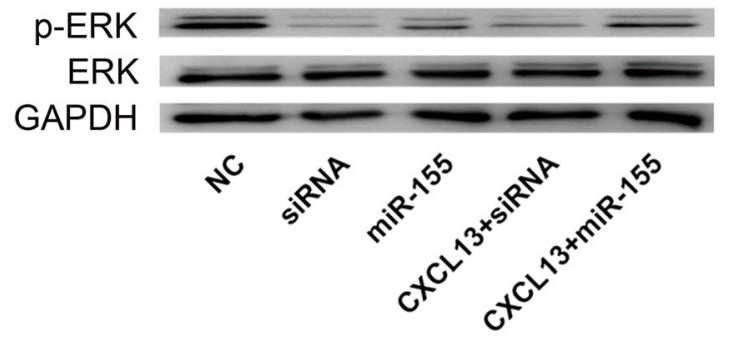

a
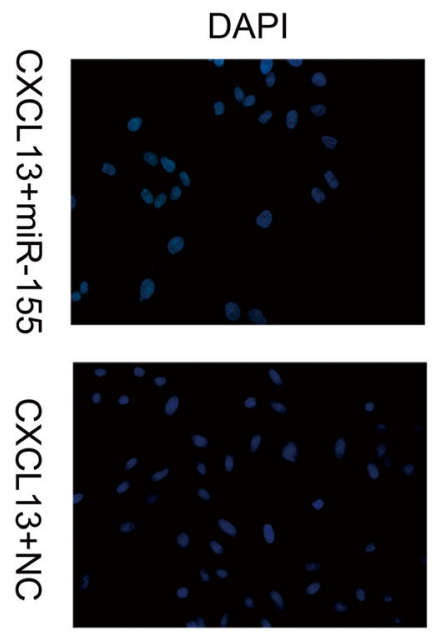

TGF- $\beta 1$

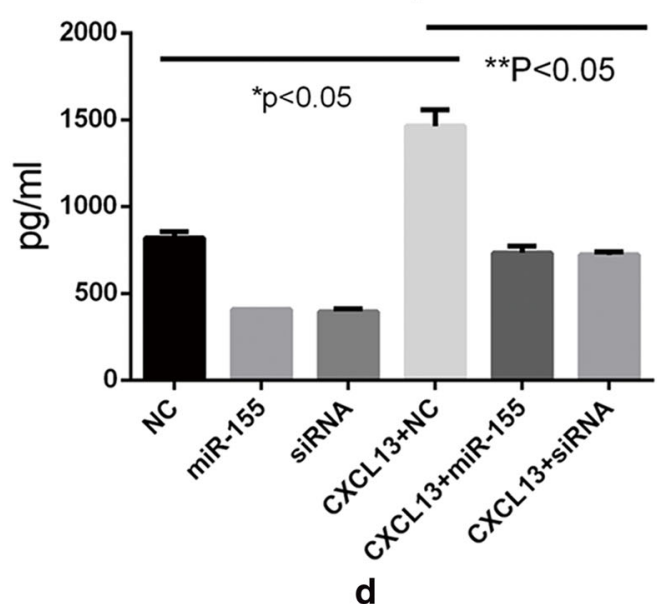

Fig. 5. Phosphorylation of ERK in HMRCs and measurement of the concentration of TGF- $\beta 1$ in supernatants of HRMCs using ELISA. (a-c) Western blot and immunofluorescent analysis in HRMCs transfected with siRNA or miR-155 mimic with or without CXCL13 treatment. (b) Relative expression of CXCR5 to GAPDH was calculated. (c) Immunofluorescent measurement of the tyrosine phosphorylation level in ERK (red). (d) The HRMCs were incubated for $12 \mathrm{~h}$ after treatment, and the supernatants were then collected for detecting TGF- $\beta 1$ levels. Statistical analyses were performed by ANOVA followed by Dunnett's test. $* p<0.05$ (NC versus CXCL13 + NC, miR-155, or siRNA); $* *<<0.05$ (CXCL13 + NC versus CXCL13 + miR-155 or CXCL13 + siRNA). 


\section{DISCUSSION}

MiR-155 is an important effector in immune system which could regulate innate and adaptive immune responses $[9,17]$. Increasing evidence indicates that many miRNAs such as miR-146, miR-21, and miR-155 have the ability to negatively regulate the activation of inflammatory pathways in myeloid cells, suggesting they have anti-inflammatory effects [20]. Previous studies have shown that mice with miR-155 gene deficiency have functional defects in $\mathrm{T}$ and $\mathrm{B}$ lymphocytes and other immune cells [30] Dysregulated miR155 expression can cause serious complications in the immune system. The expression of miR-155 is induced in multiple sclerosis (MS), rheumatoid arthritis (RA), and Sjögren's syndrome [28]. In contrast, in SLE patients, serum miR-155 levels were found to be decreased compared with that in healthy controls and positively correlated with estimated glomerular filtration rate (eGFR) [32]. Up to date, very few studies focused on the role of miR-155 in inflammatory responses in HRMCs. Mesangial cells, endothelial cells, and podocytes are three main cell types in the glomerulus [35]. Mesangial cells and their matrix form the central stalk of the glomerulus and interact closely with endothelial cells and podocytes [14]. Aberrant proliferation, apoptosis, and activation of mesangial cells are frequently observed in LN [27]. In this study, we found that CXCL13 increased HRMCs proliferation, while overexpression of miR-155 could abrogate the CXCL13-stimulated cell proliferation.

MiRNA-mediated gene regulation usually reduces the amount of target proteins [22]. To find the target of miR155 , we transfected miR-155 mimic into HRMCs and found the decreased levels of CXCR5. MiR-155 is a component of the inflammatory response and regulates the ERK-MAPK signaling pathway in T cells [24]. However, thus far there has been no information regarding the relationship between ERK and miR-155 in HRMCs. P-ERK can phosphorylate a wide range of cellular substrate, modulates the transcriptional activity of the cell, and triggers cell growth and differentiation $[13,21]$. We transfected a miR-155 mimic or siRNA in HRMCs and found decreased phosphorylation of ERK and inhibited cell proliferation rate. These results suggest that the decrease in p-ERK formation was due to the inhibition of CXCR5. But CXCR5 expression was only partially suppressed in miR-155 mimic-transfected HRMCs, suggesting that there may be an alternative pathway independent of miR-155 and CXCR5.

TGF- $\beta 1$ belongs to a family of cytokines involved in many physiological processes, including growth, differentiation, proliferation, tissue remodeling, and wound healing [8]. In LN, TGF- $\beta 1$ induces mesangial cells to undergo myofibroblastic activation or transition. When we treated HRMCs with CXCL13, the concentration of TGF- $\beta 1$ increased; conversely, when we transfected the miR-155 mimic into HRMCs, the level of TGF- $\beta 1$ decreased. These results indicated that CXCL13 may promote myofibroblastic activation of HRMCs, but miR-155 mimic abrogates this process.

In summary, our study has shown that miR-155 can reduce the proliferation of HRMCs and the production of TGF- $\beta 1$ by downregulating the expression of the CXCR5ERK signaling pathway upon CXCL13 stimulation. Our findings suggest that miR-155 is involved in the disease pathogenesis of $\mathrm{LN}$ and may be further validated as a new therapeutic target for treating $\mathrm{LN}$.

\section{FUNDING INFORMATION}

This work was supported by the National Natural Science Foundation of China (81601410); the Jiangsu Provincial Commission of Health and Family Planning (H201623); and the Special Fund for Clinical Medical Science and Technology of Nantong (HS2014071, HS2016003).

\section{COMPLIANCE WITH ETHICAL STANDARDS}

Conflict of Interest. The authors declare that there are no conflicts of interest.

Ethical Approval. The study was approved by the Ethics Committees of Affiliated Hospital of Nantong University. The approval number is 2017-L096.

Open Access This article is distributed under the terms of the Creative Commons Attribution 4.0 International License (http://creativecommons.org/licenses/by/4.0/), which permits unrestricted use, distribution, and reproduction in any medium, provided you give appropriate credit to the original author(s) and the source, provide a link to the Creative Commons license, and indicate if changes were made.

\section{REFERENCES}

1. Almaani, S., A. Meara, and B.H. Rovin. 2017. Update on lupus nephritis. Clinical Journal of the American Society of Nephrology 12 (5): 825-835. https://doi.org/10.2215/cjn.05780616.

2. Anders, H.J., and A.B. Fogo. 2014. Immunopathology of lupus nephritis. Seminars in Immunopathology 36 (4): 443-459. https:// doi.org/10.1007/s00281-013-0413-5.

3. Chen, S., and B.A. Smith. 2015. MicroRNA-155-deficient dendritic cells cause less severe GVHD through reduced migration and 
defective inflammasome activation. 126 (1): 103-112. https:// doi.org/10.1182/blood-2014-12-617258.

4. Connerty, P., A. Ahadi, and G. Hutvagner. 2015. RNA binding proteins in the miRNA pathway. International Journal of Molecular Sciences 17 (1). https://doi.org/10.3390/ijms17010031.

5. Da, Z., and L. Li. 2016. CXCL13 promotes proliferation of mesangial cells by combination with CXCR5 in SLE. 2016: 2063985.

6. Davidson, A., and C. Aranow. 2010. Lupus nephritis: lessons from murine models. Nature Reviews Rheumatology 6 (1): 13-20. https:// doi.org/10.1038/nrrheum.2009.240.

7. Devarapu, S.K., and H.J. Anders. 2018. Toll-like receptors in lupus nephritis. 25 (1): 35. https://doi.org/10.1186/s12929-018-0436-2.

8. Fujio, K., T. Komai, M. Inoue, K. Morita, T. Okamura, and K. Yamamoto. 2016. Revisiting the regulatory roles of the TGF-beta family of cytokines. Autoimmunity Reviews 15 (9): 917-922. https:// doi.org/10.1016/j.autrev.2016.07.007.

9. Garo, L.P., and G. Murugaiyan. 2016. Contribution of microRNAs to autoimmune diseases. Cellular and Molecular Life Sciences 73 (10): 2041-2051. https://doi.org/10.1007/s00018-016-2167-4.

10. Hafez, S.S., S. Saad Wel, and N.H. Shedid. 2014. B-cell-attracting chemokine-1 (BCA-1/CXCL13) in systemic lupus erythematosus, its correlation to disease activity and renal involvement. The Egyptian Journal of Immunology 21 (2): 23-32.

11. Hahn, B.H., M.A. McMahon, A. Wilkinson, W.D. Wallace, D.I. Daikh, J.D. Fitzgerald, G.A. Karpouzas, et al. 2012. American College of Rheumatology guidelines for screening, treatment, and management of lupus nephritis. Arthritis Care Res (Hoboken) 64 (6): 797-808. https://doi.org/10.1002/acr.21664.

12. Han, X., Y. Wang, X. Zhang, Y. Qin, B. Qu, L. Wu, J. Ma, Z. Zhou, J. Qian, M. Dai, Y. Tang, E.K.L. Chan, J.B. Harley, S. Zhou, and N. Shen. 2016. MicroRNA-130b ameliorates murine lupus nephritis through targeting the type I interferon pathway on renal mesangial cells. Arthritis \& Rhematology 68 (9): 2232-2243. https://doi.org/10.1002/art.39725.

13. Imperial, R., O.M. Toor, A. Hussain, J. Subramanian, and A. Masood. 2017. Comprehensive pancancer genomic analysis reveals (RTK)-RAS-RAF-MEK as a key dysregulated pathway in cancer: its clinical implications. Seminars in Cancer Biology. https://doi.org/ 10.1016/j.semcancer.2017.11.016.

14. Kurihara, H., and T. Sakai. 2017. Cell biology of mesangial cells: the third cell that maintains the glomerular capillary. Anatomical Science International 92 (2): 173-186. https://doi.org/10.1007/s12565-016-0334-1.

15. Lorenzen, J.M., H. Haller, and T. Thum. 2011. MicroRNAs as mediators and therapeutic targets in chronic kidney disease. Nature Reviews. Nephrology 7 (5): 286-294. https://doi.org/10.1038/ nrneph.2011.26.

16. Luan, J., C. Jiao, W. Kong, J. Fu, W. Qu, Y. Chen, X. Zhu, et al. 2018. circHLA-C plays an important role in lupus nephritis by sponging miR-150. Molecular Therapy-Nucleic Acids 10: 245253. https://doi.org/10.1016/j.omtn.2017.12.006.

17. Ma, Xiaodong, Lindsey E. Becker Buscaglia, Juanita R. Barker, and Yong Li. 2011. MicroRNAs in NF-kB signaling. Journal of Molecular Cell Biology 3 (3): 159-166.

18. Mashima, R. 2015. Physiological roles of miR-155. Immunology 145 (3): 323-333. https://doi.org/10.1111/imm.12468.

19. Mok, C.C. 2015. Mycophenolate mofetil for lupus nephritis: an update. Expert Review of Clinical Immunology 11 (12): 13531364. https://doi.org/10.1586/1744666x.2015.1087314.

20. O'Connell, R.M., D.S. Rao, A.A. Chaudhuri, and D. Baltimore. 2010. Physiological and pathological roles for microRNAs in the immune system. Nature Reviews. Immunology 10 (2): 111-122. https://doi.org/10.1038/nri2708.

21. Oh, S.E., and M.M. Mouradian. 2018. Cytoprotective mechanisms of DJ-1 against oxidative stress through modulating ERK1/2 and
ASK1 signal transduction. Redox Biology 14: 211-217. https:// doi.org/10.1016/j.redox.2017.09.008.

22. Patel, V., and L. Noureddine. 2012. MicroRNAs and fibrosis. Current Opinion in Nephrology and Hypertension 21 (4): 410-416. https://doi.org/10.1097/MNH.0b013e328354e559.

23. Qayum, A.A., A. Paranjape, and D. Abebayehu. 2016. IL-10-induced miR-155 targets SOCS1 to enhance IgE-mediated mast cell function. 196 (11): 4457-4467. https://doi.org/10.4049/jimmunol.1502240.

24. Rouquette-Jazdanian, A.K., R.L. Kortum, W. Li, R.K. Merrill, P.H. Nguyen, L.E. Samelson, and C.L. Sommers. 2015. miR-155 controls lymphoproliferation in LAT mutant mice by restraining T-cell apoptosis via SHIP-1/mTOR and PAK1/FOXO3/BIM pathways. PLoS One 10 (6): e0131823. https://doi.org/10.1371/journal.pone.0131823.

25. Schiffer, L., K. Worthmann, H. Haller, and M. Schiffer. 2015. CXCL13 as a new biomarker of systemic lupus erythematosus and lupus nephritis - from bench to bedside? Clinical and Experimental Immunology 179 (1): 85-89. https://doi.org/10.1111/cei.12439.

26. Schwartz, N., B. Goilav, and C. Putterman. 2014. The pathogenesis, diagnosis and treatment of lupus nephritis. Current Opinion in Rheumatology 26 (5): 502-509. https://doi.org/10.1097/ bor.0000000000000089.

27. Seret, G., Y. Le Meur, Y. Renaudineau, and P. Youinou. 2012. Mesangial cell-specific antibodies are central to the pathogenesis of lupus nephritis. Clinical and Developmental Immunology 2012: 579670. https://doi.org/10.1155/2012/579670.

28. Singh, R.P., I. Massachi, S. Manickavel, S. Singh, N.P. Rao, S. Hasan, D.K. Mc Curdy, S. Sharma, D. Wong, B.H. Hahn, and H. Rehimi. 2013. The role of miRNA in inflammation and autoimmunity. Autoimmunity Reviews 12 (12): 1160-1165. https://doi.org/ 10.1016/j.autrev.2013.07.003.

29. Sun, Y., W.Z. Liu, T. Liu, X. Feng, N. Yang, and H.F. Zhou. 2015. Signaling pathway of MAPK/ERK in cell proliferation, differentiation, migration, senescence and apoptosis. Journal of Receptor and Signal Transduction Research 35 (6): 600-604. https://doi.org/ 10.3109/10799893.2015.1030412.

30. Thai, T.H., H.C. Patterson, D.H. Pham, K. Kis-Toth, D.A. Kaminski, and G.C. Tsokos. 2013. Deletion of microRNA-155 reduces autoantibody responses and alleviates lupus-like disease in the Fas(lpr) mouse. Proceedings of the National Academy of Sciences of the United States of America 110 (50): 20194-20199. https://doi.org/ 10.1073/pnas.1317632110.

31. Towler, B.P., C.I. Jones, and S.F. Newbury. 2015. Mechanisms of regulation of mature miRNAs. Biochemical Society Transactions 43 (6): 1208-1214. https://doi.org/10.1042/bst20150157.

32. Wang, G., L.S. Tam, E.K. Li, B.C. Kwan, K.M. Chow, C.C. Luk, P.K. Li, and C.C. Szeto. 2010. Serum and urinary cell-free miR-146a and miR-155 in patients with systemic lupus erythematosus. The Journal of Rheumatology 37 (12): 2516-2522. https://doi.org/ 10.3899/jrheum. 100308 .

33. Wang, B., J.C. Jha, S. Hagiwara, A.D. McClelland, K. JandeleitDahm, M.C. Thomas, M.E. Cooper, and P. Kantharidis. 2014. Transforming growth factor-beta1-mediated renal fibrosis is dependent on the regulation of transforming growth factor receptor 1 expression by let-7b. Kidney International 85 (2): 352-361. https://doi.org/10.1038/ki.2013.372.

34. Wang, H., J. Wang, and Y. Xia. 2017. Defective suppressor of cytokine signaling 1 signaling contributes to the pathogenesis of systemic lupus erythematosus. Frontiers in Immunology 8: 1292. https://doi.org/10.3389/fimmu.2017.01292.

35. Yung, S., and T.M. Chan. 2017. Anti-dsDNA antibodies and resident renal cells - their putative roles in pathogenesis of renal lesions in lupus nephritis. Clinical Immunology 185: 40-50. https://doi.org/ 10.1016/j.clim.2016.09.002. 